\title{
Sostenibilidad de los proyectos pecuarios de la región Ch'orti' de Chiquimula, Guatemala
}

\author{
Sustainability of livestock projects in \\ the Ch'orti' region of Chiquimula, Guatemala
}

\begin{abstract}
Como citar el artículo
Linares Díaz, A. J. . Sostenibilidad de los proyectos pecuarios de la región Ch'orti' de Chiquimula, Guatemala.
\end{abstract} Revista Naturaleza, Sociedad Y Ambiente, 8(1). DOI: https://doi.org/10.37533/cunsurori.v8i1.58

Alejandro José Linares Díaz

Centro Universitario de Oriente - CUNORI - USAC

Recibido: 23 de marzo de 2021 / Aceptado: 26 de mayo de 2021

Disponible en internet el 10 de octubre de 2021

*Autor para correspondencia, correo electrónico: ajld1979@cunori.edu.gt

\begin{abstract}
Resumen
Guatemala, experimenta una condición de inseguridad alimentaria y nutricional, acentuada por los altos índices de pobreza y pobreza extrema multidimensional en la población, principalmente en áreas rurales. Uno de los departamentos más afectados por este problema es Chiquimula en su región Ch'orti', compuesta por cuatro municipios: Jocotán, Camotán, San Juan Ermita y Olopa que, albergan a más de 160,000 habitantes.

Ante este problema, la incorporación de proyectos pecuarios, ha buscado mejorar las condiciones de seguridad alimentaria y nutricional de las familias vulnerables, al proveer alimentos de diversas especies animales con alto valor nutricional, especialmente de tipo proteico. Sin embargo, a través de varias décadas, estos sistemas productivos se han reducido o perdido, ya sea parcial o totalmente y su persistencia ha sido comprometida, sin conocerse las causas.

Medir la sostenibilidad de éstos, fue el punto de partida de la presente investigación descriptiva con un enfoque mixto donde, a través de la metodología de "Marco para la Evaluación del Sistema de Manejo de recursos naturales incorporando Indicadores de Sostenibilidad" - MESMIS -, bajo el método hipotético-deductivo, en siete atributos: productividad, estabilidad, confiabilidad, resiliencia, adaptabilidad, equidad y autosuficiencia, distribuidos en 16 macroindicadores y 65 indicadores específicos, construidos especialmente para este estudio.
\end{abstract}

Palabras clave: Sostenibilidad, proyecto pecuario, seguridad alimentaria y nutricional, Ch'orti'

\section{Abstract}

Guatemala, faces a condition of food and nutrition insecurity, accentuated by the high rates of multidimensional poverty and extreme poverty in the population, mainly in rural areas. One of the departments most affected by this problem is Chiquimula, which faithfully represents this social conflict in its Ch'orti' region, made up of four municipalities: Jocotán, Camotán, San Juan Ermita and Olopa, where more than 160,000 population live.

Because of this problem, the incorporation of livestock projects stands out, whose primary purpose is to improve the conditions regarding food and nutritional security for vulnerable families, by providing food from various animal species with high nutritional value especially of a protein type. However, over several decades, these production systems have been reduced or lost, partially or totally, and their persistence has been compromised, without knowing the reasons.

Measuring their sustainability was the starting point of this descriptive research with a mixed approach where, through the methodology of "Framework for the Evaluation of the Natural Resources Management System incorporating Sustainability Indicators" - FEMSSI -, analyzed under the hypothetical-deductive method, the livestock projects implemented in the Ch'orti' region of the department of Chiquimula in the period $2008-2018$, in seven attributes: productivity, stability, reliability, resilience, adaptability, equity and self-sufficiency, distributed in 16 macro indicators and 65 specific indicators, specially created for this study.

Keywords: Sustainability, livestock project, food and nutritional security, Ch'orti' 


\section{Introducción}

Si bien, la Seguridad Alimentaria y Nutricional (SAN) no es un concepto nuevo, es una definición multidimensional que se ha utilizado de diversas maneras de acuerdo al contexto en que se presenta y que, como factor común establece que la misma, depende de la disponibilidad de alimentos que a su vez, tiene que ver directamente con la producción y estabilidad de los mismos, influenciada por las condiciones climáticas y la estacionalidad de los cultivos y que obedece sobre todo a factores económicos, de consumo y a necesidades biológicas, los cuales interactúan para establecer si lo que las personas consumen, satisfacen sus necesidades nutricionales mínimas.

La búsqueda de mejorar la situación de seguridad alimentaria y nutricional como país, ha llevado a develar la importancia de conocer tres conceptos no idénticos, pero sí ligados: seguridad alimentaria y nutricional, soberanía alimentaria y sistema alimentario. El primero se constituye en una meta a alcanzar, el segundo en el sustento de quién y para quiénes se debe conseguir y el último, en la forma de lograrlo.

De tal manera que este tercer concepto define todas aquellas actividades y actores implicados en lograr avances en la seguridad alimentaria y nutricional, determinados principalmente por la cantidad, calidad y diversidad de los alimentos a disponibilidad de la población.

Esto ha llevado al planteamiento de políticas, planes, programas y proyectos a distintos niveles que intentan solventar el problema del hambre, entre los mismos, se encuentran los proyectos agrícolas y pecuarios, puesto que a través de ellos se puede

alimentos de alto valor nutricional, sobre todo proteico, que pueden contribuir a mejorar la salud nutricional, el bienestar económico y las condiciones generales de vida de las familias vulnerables.

Esta investigación tuvo por objeto inicial, realizar un análisis de los proyectos pecuarios de la Región Ch'orti' del departamento de Chiquimula, Guatemala, pero no de forma aleatoria, sino solo aquellos que fueron concebidos bajo la justificación de mejorar las condiciones de inseguridad alimentaria que aquejan seriamente a poblaciones rurales a través de una forma válida que permita medir, evaluar y/o analizar la sostenibilidad por medio de un análisis validado y flexible, a mediano y largo plazo con una metodología que ya ha sido utilizada, generalmente para medir el parámetro en sistemas agroecológicos: "Marco para la evaluación del sistema de manejo de recursos naturales incorporando indicadores de sostenibilidad" - MESMIS -.

Esta supone que, un sistema es sostenible si posee los atributos de: productividad, estabilidad, confiabilidad, resiliencia, adaptabilidad, equidad y autosuficiencia, a través de diferentes indicadores únicos y específicos que, para el presente estudio fueron construidos para conocer si los proyectos han sido "sostenibles", de "baja sostenibilidad", o "no sostenibles", además de, establecer las causantes para dichas condiciones.

\section{Referencia Teórica}

Los datos que Guatemala presenta en términos de seguridad alimentaria y nutricional son verdaderamente preocupantes y reflejan el alto grado de vulnerabilidad del país en 
este contexto, situándola dentro de las más altas de ALC.

Ciertamente, la prevalencia de la subalimentación disminuyó de $18.1 \%$ (2000-2002) a $15.8 \%$ (2015-2017), según FAO, et al. (2018), la misma sigue estando dentro de las más altas de la región. Lo mismo ocurrió en el Índice Global del Hambre (IGH) que disminuyó de 17.3 a 15.6 del año 2000 al 2014 , según IFPRI (2015, p. 16).

Además, Guatemala es un país que produce alimentos, de hecho, según FAO; ALADI \& CEPAL (2019, párr. 2), el saldo agroalimentario para el año 2007 fue de 1.3 miles de millones mientras que para el 2016 fue de 2.3 miles de millones. El problema radica que no está disponible para todos, menos para los que ejercen una cultura de autoconsumo y los niveles de exportación tienen preferencia por encima del consumo nacional.

Factores como la desnutrición, los altos índices de pobreza no extrema y pobreza extrema, así como las malas condiciones de salud y saneamiento básico, la carencia de equidad de género, el poco acceso a los servicios de educación, la degradación ambiental, los factores culturales, entre otros, agudizan la problemática para lograr esta seguridad alimentaria y nutricional de forma que se garantice que, las generaciones presentes y futuras la alcancen. Esto ha llevado al planteamiento de un concepto integral denominado: "Seguridad Alimentaria y Nutricional Sostenible".

De acuerdo al Informe del Índice de Pobreza Multidimensional (IPM) reportado por el Ministerio de Desarrollo Social para finales del 2018, el $61.6 \%$ de la población guatemal- teca son multidimensionalmente pobres, con una incidencia en el área rural de casi el doble con respecto al área urbana $(82.5 \% \mathrm{y}$ $40.3 \%$, respectivamente), sin embargo, el área urbana y rural padecen de una pobreza de intensidad similar, difiriendo en el tipo de privaciones que sufren.

La interacción pobreza y ruralidad es marcada dado que, seis de cada diez pobres multidimensionales del país viven en el área rural $(67.6 \%)$. En cuanto al aspecto de seguridad alimentaria y nutricional, un $34 \%$ de la población se encuentra en pobreza multidimensional y además sufre de inseguridad alimentaria moderada o severa, lo que implica una combinación que va desde sacrificar una cantidad o calidad de lo que come hasta sufrir una condición de hambre (MIDES, 2019, pp. 24-29).

La búsqueda de mejorar la situación de seguridad alimentaria y nutricional a nivel global y como país, ha llevado a develar la importancia de conocer tres conceptos no idénticos, pero sí ligados: seguridad alimentaria y nutricional, soberanía alimentaria y sistema alimentario. El primero se constituye en una meta a alcanzar, el segundo en el sustento de quién y para quiénes se debe conseguir y el último, en la forma de lograrlo. De tal manera que este tercer concepto define todas aquellas actividades y actores implicados en lograr avances en la seguridad alimentaria y nutricional, determinados principalmente por la cantidad, calidad y diversidad de los alimentos a disponibilidad de la población.

Esto ha llevado al planteamiento de políticas, planes, programas y proyectos a distintos niveles que intentan solventar el problema del hambre, entre los mismos, se encuentran 
los proyectos agrícolas y pecuarios, puesto que a través de ellos se puede proveer alimentos de alto valor nutricional, sobre todo proteico, que pueden contribuir a mejorar la salud nutricional, el bienestar económico y las condiciones generales de vida de las familias vulnerables.

En Guatemala son numerosas las poblaciones que no tienen acceso a recursos que garanticen un sustento diario mínimo, resultando en una condición de inseguridad alimentaria que apremia a gran parte del país.

Uno de los mejores ejemplos de este escenario, es el pueblo Maya Ch'orti' cuya historia social ha sido tan dura y compleja que en palabras de Palma Murga (2013) representa los rasgos que definen su rostro actual: "rural", "indígena" y "pobre" (p. 295) y que, si bien el problema no es para nada nuevo en la región, ya que los primeros informes de la situación de hambruna Ch'orti' iniciaron alrededor de los años 1975-1977, fue hasta en el 2001 que la situación tomó mayor fuerza y conocimiento general en la población, lo que generó rápidamente diversas noticias en los medios de comunicación del país y posteriormente a nivel internacional.

La consecuente disminución en el acceso a alimentos y un estado de desnutrición fuerte en niños, mujeres y ancianos en el área era de esperarse, dando lugar en términos utilizados por FLACSO Guatemala (2014): a un asunto de "Conflictividad social", haciendo referencia a la inseguridad alimentaria.

Diversas formas de humanitarismo contra el hambre fueron llevados a los pobladores, con el arribo de diversos mecanismos de intervención de todo tipo y condición (Mariano, 2014). Esto sirvió de plataforma para que proyectos pecuarios, con apoyo gubernamental, no gubernamental, privado, público, nacional o extranjero, se implementarán en Guatemala (incluido el pueblo Ch'orti'), justificados en mejorar las condiciones de seguridad alimentaria y nutricional bajo una connotación de desarrollo social.

Bajo este contexto, la producción animal ha construido un pilar sobre el cual se forjó una posible solución o al menos una mejora al problema de inseguridad alimentaria de la región, siempre que la misma sea de forma sostenida, apegada a la búsqueda de un beneficio social y general y no de carácter individual, sin perder el enfoque con el que se crearon los sistemas productivos pecuarios: ser eficientes.

La intervención con programas de este tipo ha sido cada vez mayor, por medio de la crianza de conejos, pelibueyes, codornices, pollos de engorde y gallinas ponedoras, bajo un discurso de la bondad de las especies en cuanto a su facilidad de manejo y alimentación, así como el aporte de productos y subproductos de los animales y la posible venta del excedente a otras comunidades o venta de crías para obtener mayores ingresos.

Para los ch'ortíes, la presencia de animales en casa es un aspecto inherente a su historia, tal como cita Palma Ramos (2001, p. 110) en una narrativa de una mujer artesana de 22 años: "A mí me gusta trabajar la tierra, cuidar chumpes (pavos) y cuidar gallinas. No me gusta aprender otras cosas [...] hay animales y hay ganado que tengo que cuidar, porque tengo una novilla, dos toros, cuatro chumpes y cinco gallinas [...]". Esto evidencia que, están acostumbrados a trabajar con anima- 
les, pero no todos tienen las mismos tipos y cantidad de especies.

El carácter eminentemente agropecuario de la población económicamente activa nacional, se conceptualiza en la región rural oriental del país, principalmente con explotaciones familiares, normalmente con animales criollos (nacidos y adaptados al lugar, con o sin características zoomorfológicas definidas), en sistemas extensivos, con poca tierra (0.35 Mz/unidad productiva), según Peláez \& Juárez (2017, pp. 4-5), poco tecnificados, con bajos índices productivos resultado del manejo y alimentación deficiente.

Probablemente, son varios los problemas que han hecho que los proyectos pecuarios implementados en regiones aquejadas por hambruna, no tengan los efectos deseables en cuanto al impacto social a mediano y largo plazo. Por eso, transformar un sistema productivo por otro conlleva a diversos cambios y quizás las rutas tomadas han sido parcialmente erradas y los resultados no son lo que se esperaba socialmente.

\section{Metodología}

A partir del enfoque de sostenibilidad planteada en el Informe Brundtland donde define bajo una óptica antropocéntrica al desarrollo sostenible como aquel que satisface las necesidades de la generación presente sin comprometer a las generaciones futuras a satisfacer sus propias necesidades, se le ha concebido bajo criterios muy amplios que incluyen desde propuestas de análisis muy simplistas hasta evaluaciones más categóricas y concretas que permiten validar el concepto de forma más efectiva.
La multi-dimensionalidad del concepto sostenibilidad vuelve complejo su análisis, pero a la vez flexible, puesto que no existen criterios universales o fijos para su abordaje y en cada caso dependerá de tener indicadores claros y definidos que permitan comprender sin ambigüedad la sostenibilidad de un proyecto.

Por ejemplo, para Torres Lima, et al. (2004, p. 111), la sostenibilidad coloca en un mismo plano el medio ambiente y el desarrollo socioeconómico como parte de una misma realidad. Barrezueta $(2015$, p. 35$)$, resalta que para comprender las propuestas metodológicas y estructuras de un estudio de sostenibilidad agropecuario se debe enfocar el análisis de las dimensiones social, económico y ambiental.

De tal manera, que está investigación requirió, un enfoque mixto, con análisis simultaneo, predominantemente cualitativo que, integra datos cuantitativos con el fin de explicar de forma más objetiva los datos, a través de un modelo recomendado para sistemas agropecuarios, enmarcado dentro del desarrollo sostenible, como son los Marcos de Evaluación de la Sostenibilidad (MES).

Ésta investigación se contextualizó en una categoría descriptiva, dentro del análisis mixto predominantemente cualitativo, con un enfoque cíclico, sistemático y multiescalar, que de forma dinámica y específica a un determinado contexto socio-ambiental, se analiza, de forma participativa, a través de la interacción de múltiples enlazados.

La pesquisa se enmarca en un método hipotético-deductivo, donde se parte de una teoría previa y que, a través de una forma deductiva, tomando en cuenta que los atribu- 
tos o variables por separado, como independientes pero complementarios y que en conjunto provienen de un concepto mayor, se fundamentó la hipótesis principal de investigación relacionada al análisis de la sostenibilidad total de los proyectos pecuarios con enfoque en seguridad alimentaria y nutricional implementados en la región Ch'orti'.

Los datos se recogieron directamente de 145 entrevistas realizadas a pobladores beneficiarios directos (período 2008 - 2018), de proyectos pecuarios con enfoque en seguridad alimentaria y nutricional de la región Ch'orti' del departamento de Chiquimula, estratificados en los cuatro municipios mancomunados.

El instrumento utilizado, tomo en cuenta 16 macroindicadores (separados en 65 indicadores estratégicos) para los siete atributos planteados en la metodología MESMIS (a) productividad; b) estabilidad, c) confiabilidad d) resiliencia; e) adaptabilidad; f) equidad; y g) autosuficiencia o autogestión), bajo diferentes unidades de medición (porcentajes, valores, índices, datos cuantitativos y criterios cualitativos), en escala estandarizada o de valores de juicio, que representaron el valor de los indicadores con relación a una situación deseable y técnicamente correcta, definiendo condiciones máximas y mínimas (puntos críticos) para cada indicador según la recomendación de Silva \& Ramírez (2016, p. 127).

Para el presente caso, cada indicador, recibió una asignación de escala de valor de 1 a 5 , de acuerdo a recomendación de Sarandón (2002, p. 408), lo que permitió organizar la información y convertirlos finalmente a un valor unificado de criterio de diagnóstico y de sostenibilidad.
Lo anterior se muestra de mejor forma en la Tabla a continuación:

\section{Tabla 1}

Variables para el análisis de la sostenibilidad de los proyectos pecuarios con enfoque en seguridad alimentaria y nutricional de la región Ch'orti' del departamento de Chiquimula, Guatemala.

\footnotetext{
\begin{tabular}{cc} 
VARIABLE & DEFINICIÓN \\
\hline
\end{tabular}

$\begin{array}{ll}\text { Productividad } & \begin{array}{l}\text { Capacidad del sistema para proporcionar el nivel requerido de bienes y } \\ \text { servicios. Identifica el valor del atributo en un periodo de tiempo específico. }\end{array}\end{array}$

Estabilidad Propiedad del sistema de tener un estado de equilibrio dinámico estable.

Capacidad del sistema de mantener su productividad o beneficios deseados en niveles cercanos al equilibrio, ante perturbaciones normales del ambiente.

Capacidad del sistema de retomar al estado de equilibrio o mantener el potencial productivo después de experimentar perturbaciones graves.

Capacidad del sistema de encontrar nuevos niveles de equilibrio, es decir, de continuar siendo productivo o brindando beneficios ante cambios a largo plazo en el ambiente. Se relaciona con la capacidad de respuesta, para hacer frente al cambio. Este atributo incluye desde aspectos relacionados con la diversificación de actividades u opciones tecnológicas hasta procesos de organización social, de formación de recursos humanos y de aprendizaje.

Adaptabilidad

Capacidad del sistema de distribuir de manera justa, tanto intra como

Equidad intergeneracionalmente, los beneficios y costos relacionados con el manejo de los recursos naturales. Capacidad del sistema de regular y controlar sus interacciones con el sistema se incluye aquil los procesos de organización y los mecanismos de sus prioridades, su identidad y sus valores.

Fuente: elaboración propia (2019), adaptado de Masera, et al. (2000, p. 20)
}

Para el caso de la presente investigación, los indicadores utilizados se basan en los atributos consignados en la metodología MESMIS, completando un total de 65 indicadores validados por Linares (2019). Cada uno de éstos, se trabajó con una escala de valorización de 1 a 5 , para totalizar un valor ideal de sostenibilidad de 325 .

Si bien no existe teóricamente, un punto de referencia para definir entre un proyecto sostenible o no sostenible, ya que dicho valor depende de la metodología utilizada para el análisis, se tomó en cuenta estudios que han utilizado la MESMIS en su análisis de sostenibilidad, como los casos reportados por Silva \& Ramírez (2016), Giraldo \& Valencia (2010), Fonseca, et al. (2016) y Linares (2019, p. 118), pudiendo establecer valores 
proporcionales de índice mayor a 228 (> 70 $\%)$ para considerar que un proyecto muestra una condición de sostenible, de $162-228$ (50 \% - $69 \%$ ) para baja sostenibilidad y menor a $162(<50 \%)$ para no sostenible, tal como se muestra en la Tabla 2.

Evidentemente, la conceptualización de las tres categorías anteriores es prioritaria y en cuanto al contexto de este estudio se definió de la siguiente manera:

- Condición de sostenible: capacidad generalizada a través del tiempo, de satisfacer las necesidades de un proyecto pecuario en todos los atributos evaluados, sin comprometer la posibilidad de cubrir necesidades futuras de la población beneficiada.

- Condición de baja sostenibilidad: capacidad parcial a través del tiempo, de satisfacer las necesidades de un proyecto pecuario en uno o varios de los atributos evaluados, sin garantía de la posibilidad de solventar necesidades futuras de la población beneficiada.

- Condición de no sostenible: incapacidad a través del tiempo, para satisfacer las necesidades de un proyecto pecuario en uno o varios de los atributos evaluados, provocando pérdida o desaparición parcial o total de los sistemas productivos inmediatos y amenazando la implementación de otros a futuro.
Tabla 2

Índices de sostenibilidad por atributo

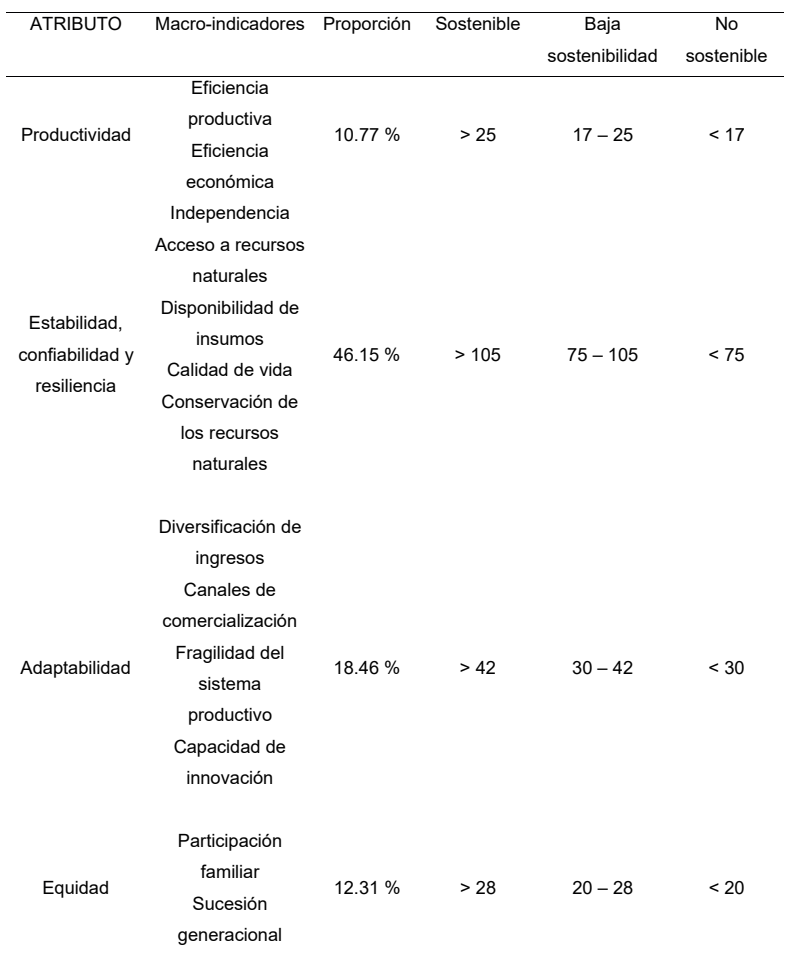

\section{Resultados}

Múltiples proyectos con un componente pecuario han sido planificados y ejecutados en la región Ch'orti' para mejorar las condiciones de hambruna y conflictividad alimentaria, con una proyección hacia el desarrollo social de la población, creando opciones que inicialmente buscan mejorar el acceso a alimentos proteicos que solventen de cierta manera la problemática nutricional de los pobres y extremadamente pobres.

Los sistemas de producción pecuaria familiar de la Región Ch'orti', por lo general, tienen una combinación de componentes tanto de agricultura como de ganadería y se establecen sobre la utilización de la mano de obra familiar, donde los hogares viven de lo que producen y de la gestión del proceso productivo, constituyéndose en un medio de capitali- 
zación para estas familias rurales, encaminados a la búsqueda de una fuente de alimentos que permita mejorar la dieta carente de proteína, pues la misma se basa en monocultivos como maíz y frijol.

Los diversos proyectos pecuarios de la región mancomunada Ch'orti' del departamento de Chiquimula, básicamente se centran en sistemas de ganado bovino, porcino, avícola, caprino, ovino, equino y especies menores como conejos, abejas y peces.

\section{Tabla 3}

Resumen del análisis de la sostenibilidad de acuerdo a los atributos evaluados en los proyectos pecuarios con enfoque en seguridad alimentaria y nutricional de la región Ch'orti' del departamento de Chiquimula, Guatemala.

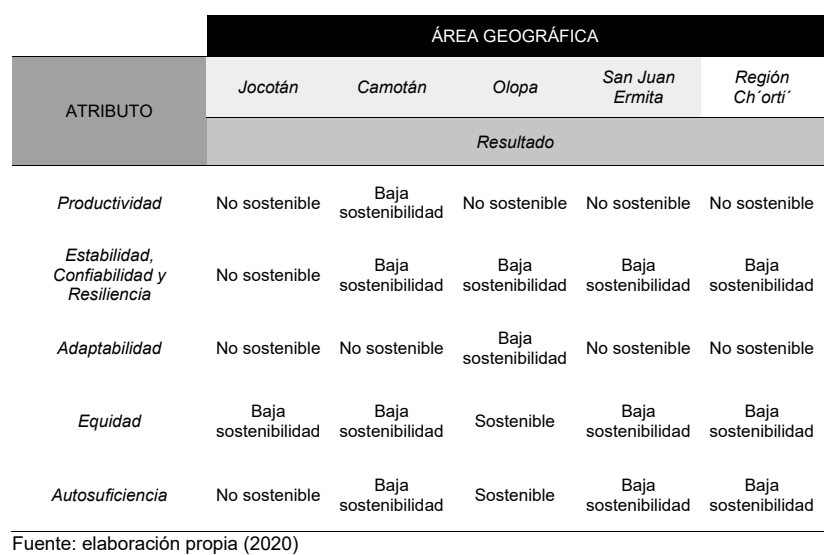

Es evidente que la condición más crítica de sostenibilidad, la tiene los proyectos examinados en el municipio de Jocotán, en virtud que mostraron ser no sostenibles para casi todos los criterios, con excepción de la Equidad, que resultó ser de baja sostenibilidad.

En el caso del municipio de Camotán, la situación es un poco mejor, pero siempre muestra una condición de baja sostenibilidad, menos en el atributo de Adaptabilidad que es no sostenible. El municipio de Olopa es el más diverso y mejor en sus resultados dado que, solamente mostró ser no sostenible en el componente de productividad, de baja sostenibilidad para Estabilidad, Confiabilidad y Resiliencia y sostenible para Equidad y Autosuficiencia. Por último, el municipio de San Juan Ermita y el promedio de la región Ch'orti', mostraron los mismos resultados, con una condición de no sostenible para los atributos de Productividad y Adaptabilidad y de baja sostenibilidad para el resto.

Si bien, los municipios mancomunados no se alejan tanto en sus valores para los parámetros considerados, si puede detectarse que, en algunos atributos como equidad y autosuficiencia, la condición del municipio de Olopa es mucho mejor al resto del área, aun cuando algunas fuentes lo aprecien en menor categoría que al resto.

Sin embargo, estas dos variables no están desligadas del resto y, por lo tanto, su baja productividad, la mermada estabilidad, confiabilidad y resiliencia y la poca respuesta de adaptación de los sistemas pecuarios hacen que, no escapa a la situación general de la región y sus cifras en materia de seguridad alimentaria y nutricional, sean similares.

Para entender de mejor forma estas realidades, se realizó un desglose de los atributos a través de un gráfico tipo amiba, tal y como se recomienda para representar los resultados obtenidos a través de la metodología MESMIS. En el mismo, se compara cada uno de los municipios por medio de 16 macroindicadores y lo lejos o cerca que pudieran estar, tanto de un nivel de sostenibilidad, como de un máximo valor posible. 
Si bien a la figura se le pudiera dar una interpretación segmentada en las variables, es importante elucidar que, no se debe eximir del análisis, el hecho que, los factores confluyen y se complementan y la exegesis del todo es más fuerte que la de sus partes, dado que la sostenibilidad es un concepto de cohesión, de naturaleza integral y que se cimienta en la perspectiva de un desarrollo multidimensional que concatena aquello que puede ser soportable, viable y equitativo.

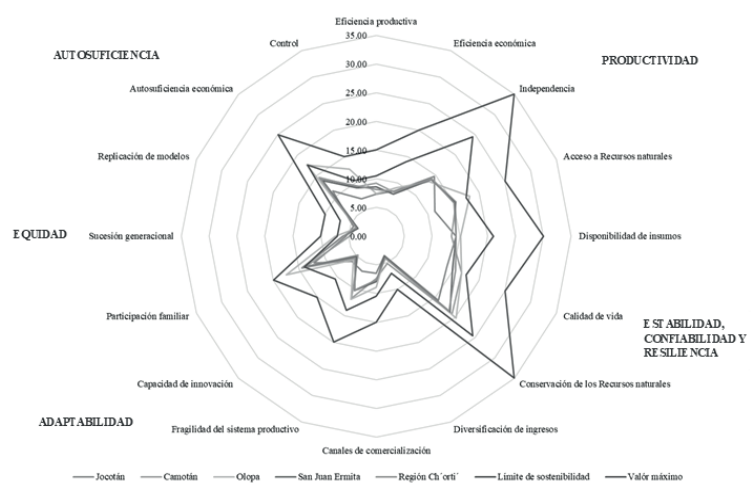

Figura 1. Representación de los macroindicadores de sostenibilidad en los proyectos pecuarios con enfoque en seguridad alimentaria y nutricional de la región Ch'orti', del departamento de Chiquimula, Guatemala.

Fuente: elaboración propia (2020)

De manera unificada, tal como se muestra en la figura 2, los proyectos pecuarios con enfoque en seguridad alimentaria y nutricional de todos los municipios ch'ortíes para el período indagado, se sitúan en una condición de baja sostenibilidad, si bien no es en todos los atributos como se describió con antelación, si en su totalidad.

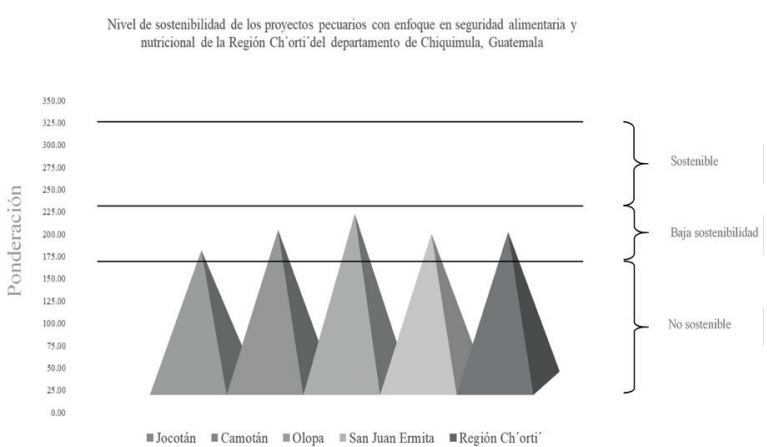

Figura 2. Nivel de sostenibilidad conjunto en los proyectos pecuarios con enfoque en seguridad alimentaria y nutricional de la región Ch'orti', del departamento de Chiquimula, Guatemala.

Fuente: elaboración propia (2020)

De esta forma, el alto grado de correspondencia entre los atributos analizados y la heterogeneidad presentada en los municipios mancomunados objetos de estudio, explican porque muchos de ellos han desaparecido y consecuentemente, sus efectos en mejorar las condiciones de seguridad alimentaria y nutricional no son notorios, al carecer de persistencia y durabilidad en la región Ch'orti'.

\subsection{Análisis por atributos}

\section{a. Productividad}

Con respecto a la productividad, los sistemas encajan en una condición de no sostenible para la Región Ch'orti' y sus municipios de Jocotán, Olopa y San Juan Ermita. Únicamente para el caso del municipio de Camotán, apenas se encuentra por encima del límite inferior de baja sostenibilidad.

Esto es resultado que, dichos proyectos producen escasamente como consecuencia de la poca cantidad de animales que poseen los beneficiarios, donde producen de forma insuficiente y principalmente, para autocon- 
sumo de carne y huevos y una mínima actividad de comercialización que apenas devuelve 20 centavos por cada quetzal invertido y si bien los períodos de producción son amplios en el año y se tiene producto en la mayor parte de los meses, no son suficientes para garantizar una rentabilidad que, haga eficientes a los proyectos.

En otros términos, se produce casi de forma permanente, pero en cantidades completamente insuficientes que no permiten cubrir las necesidades en cantidad de alimentos para los beneficiarios ni mucho menos, alcanzar niveles de rentabilidad favorables que les agencie de una prima monetaria con la que puedan comprar insumos, alimentos y otros, es decir, no cubre el factor de disponibilidad necesario para la seguridad alimentaria y nutricional, para lo cual fueron creados dichos proyectos.

\section{b. Estabilidad, confiabilidad y resiliencia}

El análisis de la estabilidad, confiabilidad y resiliencia de la región Ch'orti' a través de los indicadores planteados, identifica que en conjunto solo se alcanza un $51.56 \%$ de sostenibilidad en el área. Los municipios de Olopa, Camotán y San Juan Ermita se sitúan en una condición de baja sostenibilidad y el municipio de Jocotán como no sostenible.

Los sistemas pecuarios y condiciones generales muestran que los proyectos han sido inestables casi desde sus inicios, con baja inversión inicial y capital de trabajo, un alto grado de dependencia externa por parte de las organizaciones que introdujeron el proyecto, una incapacidad no voluntaria de los beneficiarios que les impide acceder a créditos, un uso inadecuado de los recursos naturales, imposibilita mejorar sus sistemas y adquirir cierto grado de crecimiento y tecnificación.

De hecho, pudiera considerarse que cuando un proyecto provea a los beneficiarios, de la mayor cantidad de animales posible, sumado a un seguimiento de asesoría y capacitación en el manejo y conservación de los recursos naturales, que incluya dentro de la inversión inicial cierto grado de tecnificación productivo y que además, fortalezca el proceso de gestión para posibles créditos, podrían de cierta forma, contrarrestar en algún momento el grado de dependencia externa y dar una mayor posibilidad a que los sistemas sean sostenibles. Mientras esto no cambie, seguirá siendo contrario a sus objetivos.

No obstante, es importante mencionar que, las personas manifiestan cierto grado de confiabilidad en las organizaciones intervinientes, en los sistemas pecuarios y aunque saben que no producen mucho y que probablemente los proyectos solo duraron un tiempo, consideran sentirse satisfechos con la calidad de vida que han tenido, aun cuando están conscientes que su situación de hambruna empeora gradualmente y se preocupan principalmente por su día a día, es decir, por su condición de vida a corto plazo.

Por otro lado, la información mostró que los proyectos pecuarios, mostraron una baja resiliencia ante las condiciones de vida y el cambio climático. Pocos proyectos han sabido adaptarse y los objetivos más recientes van encaminados a recuperar el material genético agropecuario de la zona, sin embargo, la poca cobertura vegetal, el uso inadecuado del suelo y agua principalmente, la falta de medidas de mitigación y conserva- 
ción de los recursos, pronostican un deterioro ambiental de crecimiento acelerado y que, por las condiciones propias de pobreza y pobreza extrema, difícilmente cambiarán de dirección.

\section{Adaptabilidad}

Para entender la adaptabilidad de un proyecto pecuario, se hace necesario entender que es una cualidad que permite ajustar un sistema a las fluctuaciones que el medio externo impone, tanto de tipo ambiental, socioeconómico, como político. Es evidente que dicha adecuación, depende directamente del nivel de especialización o tecnificación que se pudiera tener, de tal forma, que se logre responder y seguir produciendo alimento de origen animal, mediante un mejor manejo y acciones por parte de los beneficiarios.

La adaptación ambiental, induce a una valorización del entorno, de manera que los recursos naturales sean preservados y manejados de forma renovable para futuras generaciones. La adaptación social implica que el accionar de los receptores sea de beneficio colectivo, apoyando no solo a las familias inmediatas, sino también al desarrollo de la comunidad mediante la provisión de productos a costos razonables y que mejoren la provisión de alimentos para todos.

La adaptación económica, contempla un equilibrio entre producir y ser eficiente con el manejo del proyecto, con la finalidad de mejorar la cantidad y calidad de los productos, de forma tal que no solo se genere para el autoconsumo, sino además pueda comercializar una parte de lo producido.
Aclarado todo lo anterior, se determinó que la región Ch'orti' alcanza solo un $45.50 \%$ en su capacidad de adaptación con una ponderación de 27.30 de los 60.00 puntos posibles, siendo el municipio de Olopa quien presentó mejor capacidad de adaptación y una condición de baja sostenibilidad y el municipio de Jocotán, quien tuvo el menor potencial de adaptarse a cambios, tanto internos como externos al proyecto y una condición de no sostenible.

De esta manera, no existen reservas que permitan adaptarse ante los potenciales riesgos, sin posibilidad de innovar o incorporar tecnología y mucho menos afrontar los cambios medioambientales, socioeconómicos y políticos que se den en el país y que influyan en el funcionamiento de los sistemas.

\section{d. Equidad}

Es prioritario identificar que el análisis de la equidad de los proyectos pecuarios con enfoque de seguridad alimentaria y nutricional se desarrolla, tanto en la igualdad dentro de los hogares, como de éstos hacia la comunidad. No se puede concebir la idea de un proyecto sostenible, si los involucrados en el mismo, no tiene igual oportunidad de participación tanto en las obligaciones de trabajo, como en los beneficios que se generan.

De igual forma, es necesario que los favorecidos se comprometan con garantías a devolver parte del beneficio obtenido en algún grado y forma, no a la organización de intervención, sino a la comunidad misma, bajo un concepto de desarrollo social comunitario y que la mejora en las condiciones pueda alcanzar a un mayor número de familias. 
La región Ch'orti' en conjunto alcanza un $60.82 \%$ de la equidad ideal analizada, siendo Olopa el que presenta mejores condiciones con un 28.70 de los 40.00 puntos posibles y es el único que se cataloga como sostenible en cuanto a este atributo, seguido del municipio de San Juan Ermita con 24.00 y Camotán y Jocotán, con 22.50 y 22.10 , respectivamente, quedando estos, en condición de baja sostenibilidad.

Probablemente, este comportamiento obedece a cambios culturales que han influido en mayor grado en el municipio de Olopa, comparado con el resto de la región y una presencia fuerte de organizaciones de mujeres que han propiciado mejor empoderamiento del género en las actividades productivas.

\section{e. Autosuficiencia}

tEs evidente, que los beneficiarios de los proyectos, han confiado en los objetivos que los mismos procuran, buscaron cumplir siempre con las metas planteadas y están conscientes que se respeta sus conocimientos y habilidades, adaptando los sistemas a la forma de manejo que mejor les convenga, sin embargo, aunque desean que al finalizar el accionar de la organización de intervención, los módulos sean funcionales y propios, el alto grado de dependencia económica agravada con las condiciones de pobreza y pobreza extrema que caracterizan a la región Ch'orti', han complicado que sean autosuficientes.

De hecho, solamente el municipio de Olopa, apenas alcanza el límite de sostenible, los municipios de Camotán y San Juan Ermita y el promedio de la región, se catalogan como de baja sostenibilidad y el municipio de Jocotán como no sostenible.

\section{Conclusiones}

ttodos los municipios han alcanzado una condición de baja sostenibilidad, provocando la pérdida o desaparición de los proyectos en algún momento de su ciclo de vida, principalmente por su baja capacidad productiva, la inestabilidad manifiesta de los módulos ante los cambios y riesgos probables, su escasa capacidad de adaptación, la inequidad social, económica y ambiental que existe y la dependencia permanente de agentes externos para mantenerlos activos.

A esto se le puede agregar, lo poco trascendental de los mecanismos tal y como se han manejado, para aliviar la problemática de inseguridad alimentaria y nutricional, que originó este estudio.

b. Para el atributo de Productividad, los municipios han tenido una condición de no sostenibles de baja sostenibilidad. Dichas condiciones obedecen al hecho que los sistemas, no proveyeron la cantidad suficiente de animales, de acuerdo a la capacidad instalada de las familias beneficiarias, por lo que la rentabilidad de sus productos resultó por debajo de un nivel que mejorará sus ingresos de forma permanente durante el año.

c. Los aspectos Estabilidad, Confiabilidad y Resiliencia, mostraron en casi todos los municipios una condición de baja sostenibilidad, denotando que los sistemas mantuvieron una alta dependencia, sobre todo de tipo económica al estar supeditados a las organizaciones que implementaron los proyectos, principalmente porque al no existir una garantía en la provisión de los recursos naturales e insumos para el correcto funcionamiento, no hubo estabilidad, siendo causa importante en el cambio de actividades 
productivas de la población, el abandono de los módulo animales y el consecuente deterioro ambiental, retornando a la vulnerabilidad alimentaria y nutricional.

d. En casi toda la región Ch'orti', los proyectos pecuarios mostraron una condición de no sostenible con respecto a la capacidad de Adaptabilidad, dado que los sistemas incorporados para mejorar la seguridad alimentaria y nutricional de los beneficiarios, fueron demasiado frágiles, principalmente porque al ser empleados como fuentes de productos para el autoconsumo, no diversificaron los ingresos, no generaron ganancias, no se comercializaron correctamente y no permitieron el manejo de reservas para afrontar los diversos riesgos que se presentaron. A esto se añade, el poco nivel de tecnificación e innovación, derivados de la misma incapacidad de inversión que ha predominado.

e. No obstante, el atributo de Equidad, es quizás el que mejores condiciones presentó en el análisis de sostenibilidad, sigue siendo una condición de baja sostenibilidad para casi toda la región. Esto, en virtud que a pesar de la alta participación de los miembros de las familias en las actividades productivas y la elevada expectativa que los mismos se repliquen a través de las generaciones, sigue existiendo una brecha muy grande en cuanto a la igualdad de género, la toma de decisiones y la distribución equitativa del trabajo y beneficios, siendo frecuente que el padre asuma el papel de liderazgo y que solo él, determine el manejo productivo y económico.

f. En lo que respecta al atributo de Autosuficiencia, de manera general, los proyectos implementados, han adolecido de capacidad para subsistir a partir del momento en que el la institución de asistencia o cooperante, retiró el apoyo, el seguimiento y el control del mismo y que, sumado al alto grado de dependencia, principalmente económica, ocasionaron su desaparición en un período menor a dos años, posterior a la entrega completa de los sistemas, a los hogares favorecidos.

\section{Bibliografía}

Barrezueta Unda, S. (2015). Introducción a la sostenibilidad agraria: Con enfoque de sistemas e indicadores (1a ed.). Machala, Ecuador: Universidad Técnica de Machala. http://repositorio.utmachala.edu.ec/handle/48000/6928

Facultad Latinoamericana de Ciencias Sociales - FLACSO Guatemala. (2014). Las interrelaciones de la conflictividad, la inseguridad alimentaria y la migración en Guatemala. FLACSO, Guatemala. Guatemala: s. e.

FAO; ALADI; CEPAL. (2019). Perfil Nacional de Seguridad Alimentaria y Nutricional Guatemala. https://plataformacelac.org/storage/app/uploads/public/5a9/fda/209/5a9fda209c8a3566337625.pdf

FAO; WFP; OPS; OMS; UNICEF. (2018). Panorama de la seguridad alimentaria y nutricional en América Latina y el Caribe. Santiago. http://www.fao.org/3/CA2127ES/ca2127es.pdf

Fonseca, J., Cleves, J., \& León, T. (Enero-Junio de 2016). Evaluación de la sustentabilidad de agroecosistemas familiares campesinos en la microcuenca del río Cormechoque (Boyacá). Ciencia y Agricultura, 13(1), 29-47. https://revistas.uptc.edu.co/index.php/ciencia_agricultura/article/download/4804/3870/ 
Giraldo Díaz, R., \& Valencia, F. L. (2010). Evaluación de Is sustentabilidad ambiental de tres sistemas de producción agropecuarios, en el corregimiento Bolo San Isidro, Palmira (Valle del Cauca). Revista de Investigación Agraria y Ambiental -RIAA-, 7-17.

-IFPRI-, I. F. (2015). Informe de la nutrición mundial 2015: Acciones y responsabilidades para hacer progresar la nutrición y el desarrollo sostenible. Intl Food Policy Res Inst.

Linares Díaz, A. J. (Enero-Junio de 2019). Análisis de la sostenibilidad de proyectos pecuarios con enfoque en seguridad alimentaria y nutricional: la propuesta MESMIS. Perspectivas Rurales, 17(33), 85-130. revistas.una.ac.cr/index.php/perspectivasrurales/article/view/14350/19986

Mariano Juárez, L. (2014). Las hambres en la región Ch'orti' del oriente de Guatemala. Grupo 9 de Universidades. https://www.uni-g9.net/uploads/fil e s / L a s \% 20 h a m bres\%20en\%20la\%20regi\%C3\%B3n\%20C h\%E2\%80\%99orti\%E2\%80\%99\%20del\%2 Ooriente\%20de\%20Guatemala.pdf

Masera, O., Astier, M., \& López-Ridaura, S. (2000). Sustentabilidad y manejo de recursos naturales, el marco de evaluación MESMIS. (C. Alatorre Frenk, Ed.) México, D.F.: Grupo Interdisciplinario de Tecnología Rural Apropiada, A.C. https://www.researchgate.net/publication/299870632_Sustent $\quad a \quad b \quad$ i lidad_y_manejo_de_recursos_naturales_El _Marco_de_evaluacion_MESMIS

Ministerio de Desarrollo Social -MIDES(2019). Índice de Pobreza Multidimensional. Guatemala: MIDES/INE/MINFIN/SEGEPLAN.
Palma Murga, G. (2013). La situación actual de la población de origen maya en Guatemala. (M. A. Charlotte, \& A. Breton, Edits.) Guatemala, Guatemala: Asociación para el Avance de las Ciencias Sociales (AVANCSO) y Escuela de Ciencia Política, Universidad de San Carlos de Guatemala. www.mesoweb.com/publications/MMS/19_Palma.pdf

Palma Ramos, D. A. (2001). Así somos, así vivimos: Ios Ch'orti'. Guatemala: URL.

Peláez Ponce, A. V., \& Juárez Arellano, H. (2017). Lineamientos estratégicos para el fortalecimiento de la agricultura familiar y la inclusión en Guatemala. Proyecto Insignia. Guatemala: IICA. http://repositorio.iica.int/bits trea m/11324/2741/1/B VE17048772e.pdf

Sarandón, S. J. (2002). El desarrollo y uso de indicadores para evaluar la sustentabilidad de los agroecosistemas. En L. P. Ediciones Científicas Americanas (Ed.), Agroecología, El camino hacia una agricultura sostenible (págs. 393-414). Córdoba, Argentina. https://wp.ufpel.edu.br/consagro/fil e s / $2010 / 10$ / S A R A N DON-cap-20-Sustentabilidad.pdf

Silva Santamaría, L., \& Ramírez Hernández, O. (Enero-junio de 2017). Evaluación de agroecosistemas mediante indicadores de sostenibilidad en San José de las Lajas, Provincia de Mayabeque, Cuba. Luna Azul(44). http://www.scielo.org.co/pdf/luaz/n44/n44a08.pdf 
Torres Lima, P., Rodríguez Sánchez, L., \& Sánchez Jerónimo, Ó. (2004). Evaluación de la sustentabilidad del desarrollo regional. El marco de la agricultura. (E. C. Sonora, Ed.) Región y Sociedad, XVI(29), 109-144. http://www.scielo.org.mx/scieI o.php?script =sci_arttext\&pi$d=S 1870-39252004000100004$

\section{Sobre autor}

\section{Alejandro José Linares Díaz}

Zootecnista en el grado académico de Licenciatura. USAC/CUNORI. Maestro en Educación con Orientación en Medio Ambiente. USAC/CUNORI. Doctorado en Investigación para el Desarrollo Social. USAC/CUNSURORI. Asesor de Trabajos de Graduación en grado de Licenciatura en temas de: Pastos y Forrajes, Microbiología animal y de la carne, Inocuidad de los alimentos y Cunicultura. Investigador proyecto CRIA/IICA Región Oriente. Cadena Bovinos de Doble Propósito. CATIE-IICA-USDA. Delegado representante del Centro Universitario de Oriente ante el programa de Investigación en Alimentación y Nutrición de la Dirección General de Investigación - DIGI/USAC. Profesor y Asesor del curso de Investigación en la Maestría en Formulación y Evaluación de Proyectos con énfasis en Impacto Ambiental. USAC/CUNZAC. Artículos científicos publicados en la Revista del Sistema de Estudios de Postgrado -SEP- de la USAC, Revista Perspectivas Rurales de la Universidad Nacional de Costa Rica, Revista Multidisciplinaria CUNORI/USAC y Revista Científica CUNZAC/USAC

Copyright (c) 2021 Alejandro José Linares Díaz

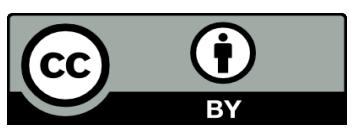

Este texto está protegido por una licencia Creative Commons $\underline{4.0}$.

Usted es libre para Compartir — copiar y redistribuir el material en cualquier medio o formato- y Adaptar el documento — remezclar, transformar y crear a partir del material- para cualquier propósito, incluso para fines comerciales, siempre que cumpla la condición de:

Atribución: Usted debe dar crédito a la obra original de manera adecuada, proporcionar un enlace a la licencia, e indicar si se han realizado cambios. Puede hacerlo en cualquier forma razonable, pero no de forma tal que sugiera que tiene el apoyo del licenciante o lo recibe por el uso que hace de la obra.

$\underline{\text { Resumen delicencia - Textocompletodelalicencia }}$

Revista digital: ISSN 2707-9643

Revista impresa: ISSN 2313-786X

Editorial Servi Prensa, Guatemala

https://doi.org/10.37533/cunsurori.v8i1.66
Vol. 8 No. 1

Enero - Diciembre

2021 
